\title{
Apropiación del espacio con agricultura: un estudio de aprendizaje asociativo mediante un modelo de simulación basado en agentes
}

\author{
Álvaro Vélez Torres \\ Universidad Autónoma Chapingo. Centros Regionales Universitarios \\ aibem@hotmail.com \\ Ricardo David Valdez Cepeda \\ Irineo Lorenzo López Cruz \\ Universidad Autónoma Chapingo \\ vacrida@hotmail.com; ilopez@correo.chapingo.mx
}

\section{Resumen}

Las formas de apropiación de un espacio siguen patrones de aprendizaje condicionado. Se sostiene que la forma que siguen estos patrones está sesgada por el aprendizaje adquirido en ensayos sucesivos. En este sentido, hemos ensayado un proceso de apropiación del espacio de un área de donación que en el pasado fue destinada a albergar basura, escombros, restos de poda y eventuales incendios para quemar desechos. La apropiación se ha hecho en la colonia Lomas de Las Hilamas, del municipio de León, Guanajuato (México), con actividad agrícola, árboles frutales, árboles de sombra y juegos infantiles. En este documento exploramos la construcción de un modelo basado en agentes que dé cuenta de la apropiación del espacio como un proceso de aprendizaje condicionado, para lo cual se retoma el modelo del aprendizaje asociativo de Rescorla-Wagner. Planteamos que aunque los colonos perciban el área de donación como un espacio ajeno, gracias a un proceso de ensayos sucesivos, se puede lograr que los vecinos se apropien de él al ver que un agente también lo hace. Después de este proceso, se ha observado que los vecinos perciben el espacio como propio y que se ha reducido su0 ocupación con basura. Los resultados del modelo de simulación basado en agentes usan una tasa de aprendizaje de 0,84 , y hemos obtenido que de promedio dos agentes se apropian del espacio 121 veces en un tiempo discreto de un año. Dichos resultados son similares a los obtenidos en los trabajos de campo.

Palabras clave: neurociencia; aprendizaje asociativo; espacio ajeno; complejidad; sistemas complejos 
Abstract. Appropriation of space with agriculture: a study of associative learning through a simulation model based on agents

The forms of appropriation of space follow conditioned learning patterns. The way these patterns follow is held to be biased by learning acquired in successive trials. In this sense, we have tested a process of appropriation of the space of a donation area that in the past was appropriated with garbage, with rubble, with pruning remains and with eventual fires to burn the garbage. The appropriation has been with agricultural activity, with fruit trees, with shade trees and with children's games in the Lomas de Las Hilamas neighborhood of the municipality of León, Guanajuato, Mexico. In this document, we explore the construction of a model based on agents that account for the appropriation of space as a conditioned learning process, for which the Rescorla-Wagner associative learning model is taken up again. We propose that if the donors perceive the donation area as a foreign space through a process of successive tests of exposure to an appropriate agent of the space, it can be achieved that other neighbors also appropriate the space. After this process, the residents have been seen to perceive the space as their own, and the appropriation with garbage has been reduced. The results of the agent-based simulation model use a learning rate of 0.84 and we have obtained that on average 2 agents appropriate the space 121 times in a discrete time of one year. These results are similar to those specified in the field work.

Keywords: neuroscience; associative learning; alien space; complexity; complex systems

\section{Sumario}

1. Introducción 6. Conclusiones

2. Método Agradecimientos

3. Resultados Financiación

4. Resultados del modelo Referencias bibliográficas

5. Análisis y discusión Anexo 1. Código Netlogo

\section{Introducción}

El concepto de apropiación del espacio puede ser definido como la totalidad de las acciones a las que procedemos en la transformación de nuestro entorno con un cierto fin (Noschis et al., 1979. Dicho proceso está fuertemente influenciado por las significaciones del espacio apropiado, independientemente de su propiedad legal (Vidal et al., 2004; Vidal y Pol, 2005). En este documento, para acotar el sentido de espacio, suponemos, como sugiere Fraassen (1970) que decir que las cosas están ubicadas en el espacio indica que tienen alguna relación entre sí. Del mismo modo, decir que las cosas suceden en el tiempo implica que suceden en cierto orden (Fraassen, 1970). De esta manera, entendemos el espacio como el orden que asignamos arbitrariamente a las cosas, y el tiempo, como la secuencia que describe dicho orden. Además, aceptamos que los fenómenos existen en espacios y tiempos simultáneos. De esta forma, el espacio no es un elemento físico concreto de la realidad, sino que es una construcción social interpretada, es una condición de posibilidad 
(Faragó, 2016). Con respecto a esto, consideramos que la construcción del espacio puede ser interpretada como un proceso de aprendizaje y, como todo proceso de aprendizaje, puede llegar a ser inconsciente. Por ejemplo, ajustamos inconscientemente nuestro comportamiento a medida que nos movemos de una ciudad a otra (Fontana-Giusti, 2016).

De esta manera, si consideramos que un conjunto $\pi>1$ de personas ha adquirido un aprendizaje sobre cómo debe apropiarse del espacio en diferentes situaciones, podemos considerar que una vez presentes tales situaciones las personas se apropiarán del espacio de forma inconsciente. Sin embargo, dicho aprendizaje no es una piedra monolítica e inmóvil. Por el contrario, podemos considerar que el inconsciente colectivo ${ }^{1}$ es transformable, porque la idea, la visión y la percepción del espacio también están abiertas a la recalibración, la reeducación y la transformación (Loukaki, 2014).

En este documento se recurrió al modelo del aprendizaje asociativo propuesto por Rescorla-Wagner (1972). ${ }^{2}$ Por un lado, dicho modelo permite estudiar procesos de aprendizaje en la apropiación del espacio y, por otro, modificarlos. El supuesto básico del modelo se basa en el circuito del miedo. Dicho circuito explica que mediante un proceso de anticipación los organismos aprenden y se adaptan a los cambios del ambiente (Leher, 2009). De esta manera, si el organismo anticipa con éxito el resultado de un fenómeno, su cerebro libera dopamina y, en consecuencia, experimenta una sensación de felicidad (Leher, 2009). Por el contrario, si no anticipa con éxito un fenómeno, su cerebro no libera dopamina y experimenta una sensación de disgusto y malestar (Leher, 2009). En la vida real, no anticipar el resultado de ciertos fenómenos puede causar la muerte de los organismos, por lo que el miedo a no anticipar el resultado se traduce en un mecanismo de aprendizaje (Leher, 2009). En consecuencia, en el modelo de Rescorla-Wagner (1972), ${ }^{3}$ el aprendizaje ocurre cuando en un ensayo la expectativa del organismo no coincide con lo sucedido (Wilson, 2012). En otras palabras, cuando un organismo predice el flujo de una acción, su aprendizaje es cero (Wilson, 2012). Por el contrario, cuando no puede anticipar el resultado, el organismo aprende (Wilson, 2012).

En este contexto, desde la década de los ochenta el ascenso de las emociones a la esfera científica ha enfatizado el papel de las emociones en la toma de decisiones racionales (Lehrer, 2009). Es decir, cómo se relacionan los procesos de aprendizaje con la toma de decisiones. De lo anterior, podemos preguntar: ¿cómo funciona el cerebro emocional cuando tomamos decisiones? Grosso modo, la toma de decisiones es un proceso de predicción y adaptación (Lehrer, 2009). La predicción se asocia a la dopamina, una hormona relacionada con el placer, con sensaciones agradables y con las formas más viscerales de disgusto (Lehrer, 2009). Por ejemplo, si se enseña a un mono a esperar jugo de frutas después de cierta secuencia de eventos, sus células de dopamina supervisan

1. De los aspectos geográficos del espacio.

2. En Black y Prokasy (1972).

3. En Wilson (2012). 
cuidadosamente la situación. Si todo va bien según lo previsto, sus neuronas dopaminérgicas secretan un pequeño estallido de placer y el mono está feliz (Lehrer, 2009). Pero si las expectativas no se cumplen, si después de cierta secuencia de eventos el mono no obtiene el jugo de frutas que anticipó, sus células de dopamina entran en huelga e inmediatamente envían una señal anunciando el error y dejan de liberar dopamina; en este caso, el mono no está feliz (Lehrer, 2009).

Por otro lado, la adaptación se asocia a la novedad (Lehrer, 2009). Para esto, el cerebro amplifica el impacto de las predicciones equivocadas y cada vez que experimenta algo inesperado la corteza cerebral inmediatamente se da cuenta (Lehrer, 2009). De esta manera, cuando las neuronas de dopamina hacen una predicción errónea, las células de la corteza cingulada anterior envían una señal eléctrica única conocida como negatividad relacionada con el error (Lehrer, 2009). En otras palabras, cada vez que se experimenta una sensación de alegría o de desilusión, de miedo o de felicidad, las neuronas están ocupadas reconectándose a sí mismas para construir una teoría de qué señales sensoriales precedieron a las emociones (Lehrer, 2009).

Estos hallazgos han sido aplicados en el plano de la simulación basada en agentes en el estudio de la emergencia de fenómenos sociales. Por ejemplo, Epstein (2013) diseñó un modelo basado en agentes que estudia por qué emergen comportamientos hostiles hacia personas de diferentes nacionalidades, incluso en personas que nunca han sido agredidas por extranjeros. En este fin, Epstein (2013) recurre al uso de tres componentes: uno emocional, uno de racionalidad limitada y uno social.

El componente emocional se basa en el modelo de Rescorla-Wagner (1972). ${ }^{4}$ Dicho modelo de aprendizaje asociativo presenta similitud con el comportamiento del cerebro emocional en la toma de decisiones racionales, como fue mencionado más arriba, principalmente en lo que se ha denominado circuito del miedo. Basándonos en lo anterior, nos propusimos hacer un estudio exploratorio respecto a la apropiación del espacio con agricultura como proceso de aprendizaje asociativo. Además, con los resultados obtenidos, diseñar un modelo de simulación basado en agente en plataforma Netlogo útil en el estudio de la apropiación espacial como aprendizaje asociativo. El objetivo de dicho modelo de simulación fue tratar de replicar un fenómeno empírico de apropiación del espacio, para que en futuros trabajos se puedan manipular los parámetros del modelo y estudiar y construir escenarios de apropiación del espacio.

El contexto en el que hemos desarrollado la investigación es urbano. Se trata de una superficie de $800 \mathrm{~m}^{2}$ que en los últimos años ha sido apropiada con basura, escombros, restos de poda, y a la que eventualmente se prende fuego para quemar los desechos y el pasto seco. Así, hemos catalogado este tipo de apropiación como apropiación del espacio ajeno. Dicha cuestión reviste interés en numerosos estudios sobre la problemática en las ciudades.

4. En Black y Prokasy (1972). 
En todo el mundo, el $54,5 \%$ de la superficie es urbana y alberga a 4 billones de personas (ONU-Habitat, 2016). En el año 2050, el $60 \%$ de la población mundial vivirá en ciudades (ONU, 2016) y la población prácticamente se duplicará (ONU, 2016), lo que significa que la población mundial será de aproximadamente 9 billones a 11 billones de personas (Mazoyer y Roudart, 2006). Esto hará de la urbanización una de las tendencias más transformadoras en el siglo xxi [...]: «Ello plantea enormes problemas de sostenibilidad en materia de vivienda, infraestructura, servicios básicos, seguridad alimentaria, salud, educación, empleos decentes, seguridad y recursos naturales» (ONU, 2016).

La colonia donde se ubica dicha área de donación se llama Lomas de Las Hilamas, del municipio de León, Guanajuato (México). Los trabajos de apropiación del espacio con agricultura comenzaron en noviembre de 2016.5 Respecto al área de donación, en los últimos años ningún vecino ha mostrado interés por este espacio pese a estar ubicado enfrente de sus hogares. Antes de comenzar la apropiación con agricultura, preguntamos a los vecinos qué opinaban del área de donación, y las respuestas fueron que era suelo del Gobierno y que no les pertenecía. Después del primer año de trabajos (en noviembre de 2017), algunos vecinos comentaron que se abstenían de participar en la apropiación del área por temor a meterse en problemas con otros vecinos, con el Gobierno o con las personas que tiran basura en el terreno de donación.

A la fecha (2018), es el segundo año que hemos sembrado en una superficie de $40 \mathrm{~m}^{2}$, hemos plantado 16 árboles frutales y 5 de sombra. Eventualmente, hemos llevado a cabo reuniones para acordar labores de limpieza. Sin embargo, el mantenimiento del área se ha restringido a dos personas. Contrariamente, después de aplicar un sondeo de opinión a los vecinos, encontramos que consideran el área como propia, que les gustaría participar en el proyecto y que no se habían integrado al proceso de apropiación por falta de tiempo o por desconocimiento.

De esta manera, cuando hacemos una analogía entre el modelo del aprendizaje asociativo y la toma de decisiones del cerebro emocional, podemos considerar que los vecinos han aprendido que la apropiación del área de donación no trae consigo una consecuencia negativa. Por ejemplo, al inicio del estudio, preguntamos a ocho personas de la colonia seleccionadas al azar cuál era su percepción del área de donación. Respondieron que esa área no era suya, que pertenecía al municipio. Posteriormente, después de implementar el proyecto de apropiación del espacio, hicimos la misma pregunta a ocho personas seleccionadas al azar y respondieron que esa área era de la colonia y que no se habían integrado al proyecto porque no tenían tiempo. Cabe mencionar que se ha respetado el área del huerto y que ha disminuido la basura en el área de donación.

Por otra parte, el modelo basado en agentes que hemos desarrollado fue alimentando una tasa de aprendizaje del 0,84 y una tasa de olvido del 0,16

5. Actualmente continuamos con el seguimiento del proyecto. 
para cada agente. ${ }^{6}$ Dichos valores se obtuvieron de una serie de ensayos realizados en un estudio de apropiación del espacio en el huerto escolar. ${ }^{7}$ Los ensayos consistieron en asignar a los niños una superficie para construir un huerto, y después de los ensayos (días) los niños se apropiaron del espacio. Los resultados fueron que en el segundo ensayo los niños delimitaron su espacio, barrieron la basura y regaron el huerto. En este sentido, establecimos que valores cercanos a 1 son los más bajos de aprendizaje y que los cercanos a 0 son los más altos. Es decir, cuando los niños anticipan que el espacio del huerto les pertenece, pueden predecir con certeza que pueden apropiárselo sin temor a que algún niño o maestro se lo impida, por lo tanto, su aprendizaje disminuye. Por el contrario, al inicio, los niños preguntaban reiteradamente si los maestros o la directora de la escuela los regañarían por apropiarse del espacio con el huerto. En otras palabras, no podían predecir con precisión qué sucedería y, por lo tanto, su aprendizaje aumentó.

De esta manera, en este caso ajustamos la velocidad de aprendizaje según la fecha observada, como sugieren Beherns et al. (2007). En nuestro caso, establecimos que en el primer ensayo los niños aprendieron que el espacio era propio, y en ensayos sucesivos reforzaron dicho aprendizaje. Así, los valores de 0,84 para la tasa de aprendizaje y de 0,16 para la tasa de olvido eran los que más nos permitían representar el fenómeno observado empíricamente. Además, inspirados en los trabajos de Black y Prokasy (1972) y de Beherns et al. (2007), pudimos observar que determinar dichos valores sigue siendo objeto de estudio. Con estos valores, los resultados simulados muestran que en un año emergerán de promedio procesos de apropiación del espacio 122 días y que el número de agentes será de dos. Es decir, que las 122 veces que se lleve a cabo un proceso de apropiación únicamente dos personas se apropiarán del espacio. Dichos datos son análogos a los obtenidos en campo. El número de personas que han llevado a cabo procesos de apropiación es de dos y, eventualmente, se ha observado una participación de tres o cuatro personas, sin embargo, en la mayoría de los casos han sido únicamente dos. Con respecto al método empleado, lo hemos denominado de seguimiento, y ha consistido en implementar la apropiación del espacio con agricultura y observar los fenómenos emergentes. Además, debido a que seguimos los principios del modelado basado en agentes, no existe un organizador de los trabajos, por el contrario, estamos centrados en observar la emergencia de patrones de abajo hacia arriba debido a que trabajamos con conjuntos de $\pi>1$ de personas.

6. Basado en trabajos de apropiación del espacio llevados a cabo en una escuela primaria de 2017 a la fecha.

7. Dichos trabajos se llevan acabo en la Escuela Primaria Ford No. 1. Se ubica en la colonia Barrio de San Miguel del municipio de León, Guanajuato (México). Los trabajos comenzaron en 2017 a la fecha. 


\section{Método}

El presente apartado está organizado de la siguiente manera. Primero, se describirá brevemente en qué consiste la técnica de modelado y simulación basada en agentes; posteriormente, presentaremos el pseudocódigo que diseñamos en el estudio de la apropiación del espacio como aprendizaje asociativo, por último, presentamos como se recabaron los datos en campo.

\subsection{Enfoque metodológico basado en agentes}

En resumen, el enfoque metodológico basado en agentes es una técnica de modelado y simulación computacional que permite brindar heterogeneidad a los agentes - unidades autónomas que representan personas o cosas (Wilensky y Rand, 2015). Todos los agentes siguen reglas sencillas de decisión, y no hay un control central de arriba abajo que supervise las acciones de los agentes. Por el contrario, las interacciones entre los agentes, así como entre los agentes y su ambiente, generan patrones recurrentes que emergen de abajo arriba cuando simulamos el modelo (Wilensky y Rand, 2015; Epstein y Axtell, 1996). Esta técnica está indicada para estudiar fenómenos complejos. Entendemos que en un sistema complejo muchas partes están interconectadas (Wilensky y Rand, 2015). De esta manera, en su estudio, el método analítico no es pertinente (Gershenson, 2007). Además, la simulación basada en agentes permite tratar de forma sencilla la complejidad, la emergencia y la no linealidad de muchos fenómenos sociales (García-Valdecasas, 2011).

\subsection{Pseudocódigo}

Siguiendo las recomendaciones de Wilensky y Rand (2015) en la construcción de un modelo basado en agentes, lo principal es identificar los mecanismos que pueden generar y explicar los fenómenos de la realidad que queremos simular. Debido a que nos interesa estudiar la apropiación del espacio como un proceso de aprendizaje asociativo, diseñamos el siguiente pseudocódigo. Anexamos código Netlogo (ver anexo 1).

1. Cree una sociedad artificial de agentes.

2. Divida en dos categorías: vecinos y apropiadores.

3. Establezca un mecanismo para que los agentes caminen.

4. Establezca un mecanismo de reforzamiento positivo con una tasa de aprendizaje de 0,84 .

5. Establezca un mecanismo de reforzamiento negativo con una tasa de extinción de 0,16 .

6. Mecanismo de aprendizaje. Si un agente de la categoría vecinos observa a un apropiador, aprende que puede apropiarse del espacio. Si su nivel de aprendizaje es mayor que 0,84 , se apropia del espacio disponible.

7. Mecanismo de extinción. Si un agente de la categoría vecinos con una tasa de aprendizaje mayor o igual a 0,84 no observa a un apropiador, 
aplica un reforzamiento negativo y su aprendizaje disminuye en 0,16 . Si su aprendizaje es menor que 0,84 , aprende que el espacio es ajeno y no se apropia de él.

8. Establezca un tiempo discreto de 1 año.

9. Simule un año.

10. Estudie la curva de agentes que aprenden a apropiarse del espacio.

Para diseñar el mecanismo de aprendizaje asociativo se usó la ecuación del aprendizaje asociativo (Rescorla-Wagner, 1972 en Wilson, 2012) ver ecuación siguiente.

$$
\Delta \mathrm{V}=\alpha \beta\left(\lambda-\sum \mathrm{V}\right)
$$

Donde:

$\Delta \mathrm{V}=$ Aprendizaje

$\alpha=$ Tasa de aprendizaje

$\beta=$ Novedad

$\lambda=$ Error de predicción

$\sum \mathrm{V}=$ fuerza asociativa entre el estímulo neutral y la respuesta condicionada

En dicha ecuación, el aprendizaje sigue una curva asintótica con valores de 0 a 1 . Donde los valores cercanos a 1 indican poco aprendizaje y los valores cercanos a 0 indican extinción del aprendizaje u/o olvido. Además, al inicio de los ensayos la fuerza asociativa entre el estímulo neutral y la respuesta condicionada adquieren valores de 0 y el error de predicción adquiere valores cercanos a 1 . Esto quiere decir que al inicio de los ensayos los organismos son incapaces de asociar el estímulo neutral con la respuesta condicionada y por lo tanto no pueden anticipar el resultado. Por otro lado, la tasa de aprendizaje es una constante que se asigna con base en evidencia empírica y cuyos valores oscilan de 0 a 1 . De esta manera, un valor cercano a 0 indica una tasa baja de aprendizaje y viceversa. En este sentido, para precisar la operatividad de la ecuación en el apartado de resultados se mostrarán un ejemplo.

Para el diseño del pseudocódigo, nos apoyamos en el método de RescorlaWagner (1972) de aprendizaje asociativo. En primer lugar, porque, como ha señalado Leher (2009), en neurociencias el circuito del miedo explica el proceso de aprendizaje adaptativo. En resumen, el aprendizaje sigue una curva asintótica con valores de 0 a 1 , donde los valores cercanos a 1 indican poco aprendizaje y los valores cercanos a 0 indican extinción del aprendizaje u olvido. En nuestro contexto, esto quiere decir que cada vez que los vecinos ven a otro vecino apropiarse del espacio con agricultura aprenden que también ellos pueden apropiárselo, sin embargo, cuando dejan de verlo, aprenden que el espacio es ajeno. Para esto se empleó una tasa de aprendizaje de 0,84 y una tasa de olvido de 0,16 . 


\subsection{Método de seguimiento}

Etapa 1 (de noviembre de 2016 a diciembre de 2017)

La primera actividad que realizamos fue hablar con los vecinos de la colonia sobre la posibilidad de llevar a cabo agricultura en el área de donación de la colonia. Posteriormente, acudimos con el presidente de colonos para solicitar permiso para llevar a cabo el proyecto. En el paso siguiente, charlamos nuevamente con los vecinos y colocamos una lona para informar de los días y los horarios en los que estaríamos trabajando en el proyecto. Luego, llevamos a cabo la siembra de frijol, maíz y calabaza en marzo. Posteriormente, hemos usado un método de seguimiento que ha consistido en observar cómo los vecinos reaccionaron a la implementación del proyecto e identificar qué fenómenos han emergido.

Etapa 2 (de enero de 2018 a diciembre de 2018)

En el siguiente año, repetimos el mismo procedimiento, es decir, hablamos con los vecinos y con el presidente de colonos, invitamos a las personas a la siembra de frijol y maíz, y por último sembramos. Cabe señalar que en este ciclo sembramos hasta el mes de mayo.

\section{Resultados}

En este documento, es importante enfatizar que el método de seguimiento que hemos presentado corresponde a un estudio de caso. Además, dicho método ha permitido transformar de manera empírica la forma de apropiación del espacio. Esto reviste interés debido a la búsqueda de soluciones de orden práctico que ayuden a construir escenarios de desarrollo en el contexto actual de crisis ambiental, social y económica, que se acentúa en los contextos urbanos. Sumado a lo anterior, dicho estudio permite hacer acercamientos teóricos entre varias disciplinas. Uno de los primeros resultados fue que a pesar de acordar una fecha para llevar a cabo los trabajos de agricultura en el área de donación, ningún vecino acudió a la cita. Posteriormente, en el transcurso del proyecto, algunos vecinos de la colonia preguntaban y ofrecían su ayuda para participar, sin embargo, no acudían a ayudar. En la figura 1, podemos ver la lona que se colocó para informar a los vecinos, así como a un integrante de la colonia.

Como mencionamos anteriormente, antes de llevar a cabo la apropiación del área de donación con agricultura, dicho espacio fue ocupado con basura y escombro (figura 2).

Debido a que el área de donación fue cubierta con tepetate, además de ser un suelo con muchas piedras, recurrimos a la siembra de frijol como mejorador de suelo. Invitamos a los vecinos a reciclar el agua de sus casas, pero solo uno se sumó a la propuesta. Para facilitar la recolección de agua reciclada, le colocamos un contenedor, sin embargo, se lo robaron.

$\mathrm{Al}$ inicio de las actividades y hasta la fecha, los niños se han acercado a ayudar, pero no los forzamos a participar, solo los invitamos a colaborar cuando vienen. 
Figura 1. Lona colocada para informar a los vecinos de la colonia

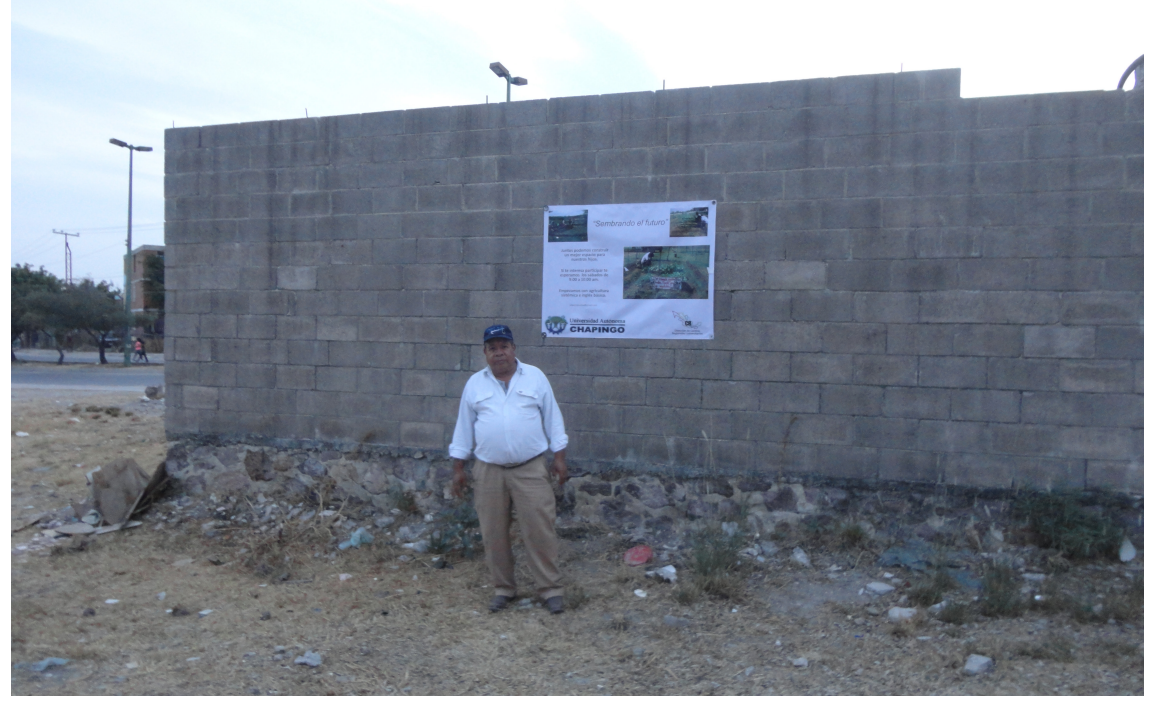

Fuente: elaboración propia.

Figura 2. Apropiación del espacio ajeno urbano con basura
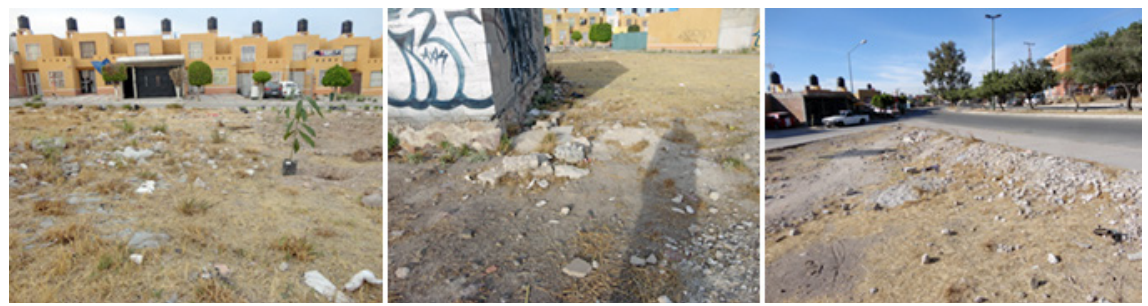

Fuente: elaboración propia.

Hasta ese momento, nosotros seguíamos con la apropiación del área de donación con agricultura y 8 árboles frutales, sin que ningún vecino se involucrara. Pero en febrero de 2017 unos guardias de seguridad de la colonia llevaron a cabo una apropiación del área de donación. Primero, recogieron la basura del área de donación y delimitaron el huerto con piedras (figura 4). Después de esto, las personas empezaron a no cruzar por el área del huerto, ya que antes observábamos continuamente pisadas en los surcos.

Posteriormente, a mediados de agosto de 2017, un vecino de una casa ubicada a un lado del área de donación trasplantó una planta de calabaza y delimitó la superficie de la planta con piedras (figura 5).

En noviembre, realizamos la cosecha y obtuvimos de promedio 3 kilos de frijol y 20 mazorcas de maíz en una superficie de $20 \mathrm{~m}^{2}$ (figura 6). No usamos 
Figura 3. Apropiación del espacio por guardias de seguridad
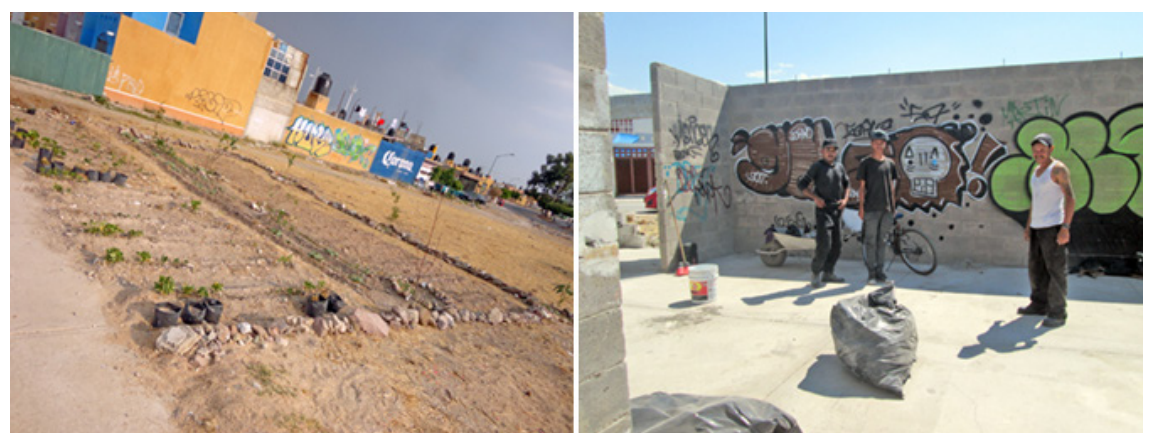

Fuente: elaboración propia.

Figura 4. Planta de calabaza trasplantada por un vecino de la casa de la barda de color verde
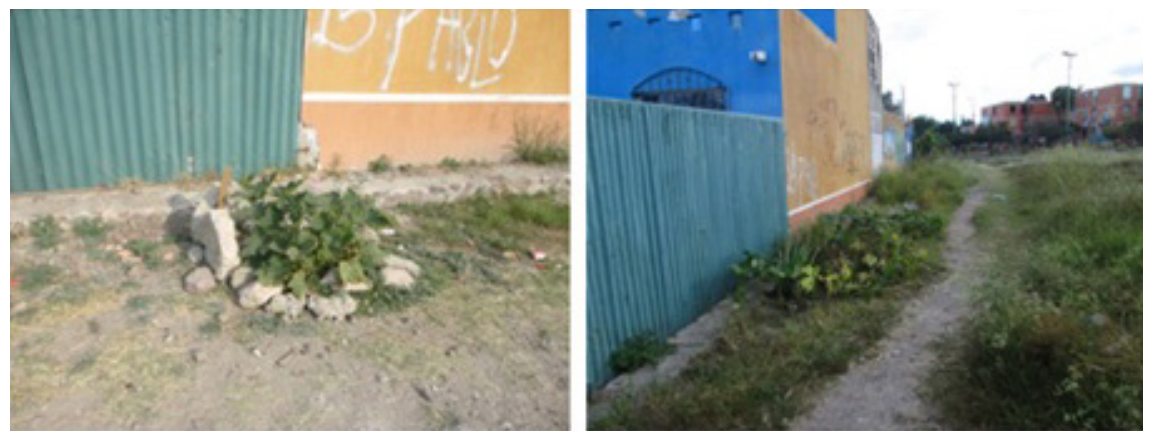

Fuente: elaboración propia.

ningún tipo de herbicidas, y el control de las plantas competidoras se hizo por medio mecánico. Guardamos la semilla de frijol obtenida la para volver a sembrar, y el maíz lo compartimos con los niños de la colonia.

Posteriormente, en diciembre de 2017 cortamos las plantas de maíz y las enterramos en el suelo para incorporar materia orgánica. En este mismo mes, colocamos un pasamanos, una banca a un costado del huerto y un par de porterías, con la intención de promover la apropiación del espacio. Además, con ayuda de un vecino, plantamos 8 nuevos árboles frutales (figura 7).

\section{Segundo año}

En esta ocasión, pospusimos la siembra hasta el mes de mayo, principalmente porque no logramos que los vecinos nos proporcionaran agua reciclada para regar el huerto. Sembramos frijol y maíz. El cuidado y el mantenimiento de los árboles corrió a nuestro cargo y al de un vecino que participó en la plantación.

En septiembre, se acordó con los vecinos hacer una limpieza del área de donación, así como llevar a cabo la plantación de 6 fresnos, árboles de 
Figura 5. Cosecha

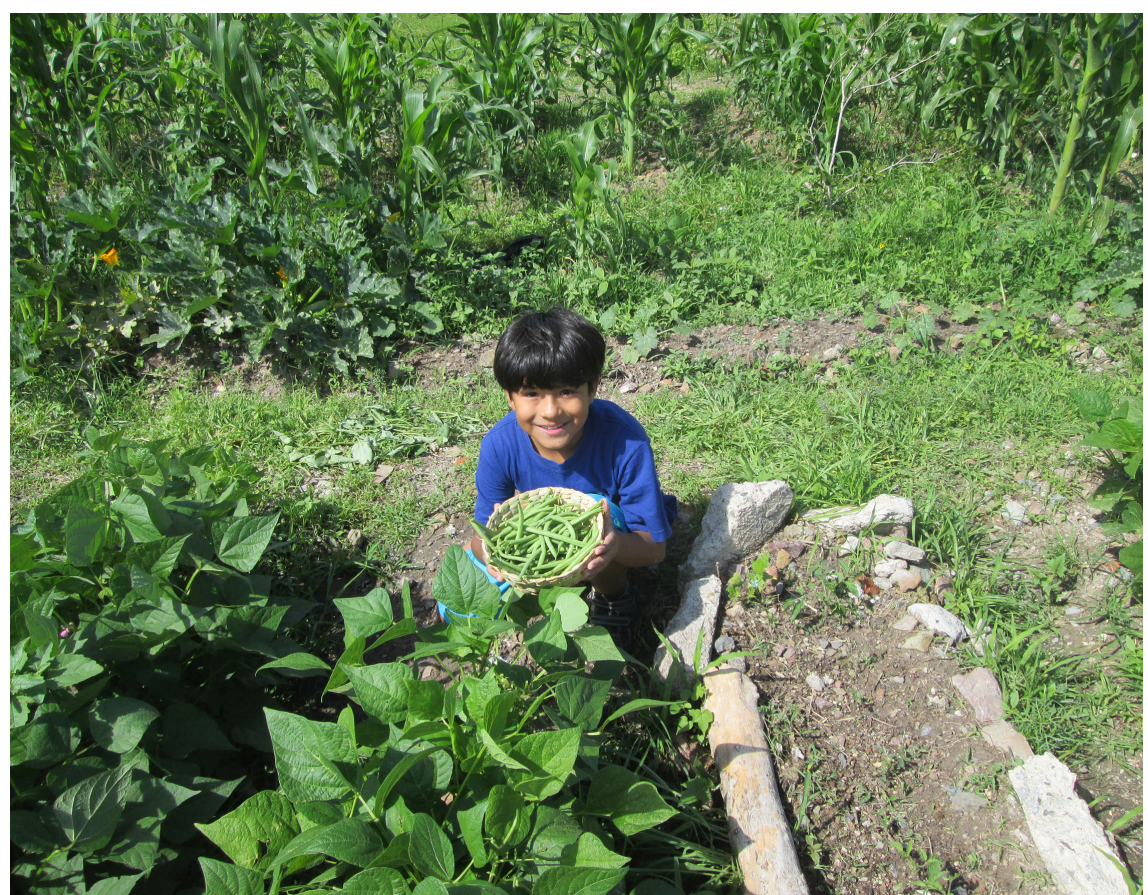

Fuente: elaboración propia.

sombra. Sin embargo, solo un vecino acudió a limpiar el área. Posteriormente, otros dos vecinos ayudaron a hacer las cepas para los árboles. Esta fue la única ocasión en que hubo más de dos vecinos trabajando en la apropiación del área (figura 8).

En el mismo mes, un grupo de niños nos solicitaron el apoyo para poner un huerto en el extremo sur del área de donación, enfrente de sus casas. Sembramos maíz, calabazas y rábanos. Sin embargo, los niños no regaron el huerto y solo prosperaron algunas plantas de frijol y de calabaza (figura 9).

Como comentamos anteriormente, colocamos un par de porterías. Sin embargo, en octubre de 2018, una fue destruida y la otra robada. Algunos vecinos comentaron que fueron niños de otra colonia, pero que nadie dijo nada por temor a agresiones por parte de las pandillas o de los padres de los niños. En dicha área, se observaba continuamente niños y adultos jugando a la pelota.

Por último, llevamos a cabo la cosecha, y en esta ocasión obtuvimos 2 kilos de frijol y 20 mazorcas de maíz. Como cosechamos en octubre, decidimos sembrar frijol nuevamente debido a la abundante precipitación de ese año. Además, en esta segunda intervención, un vecino de la colonia sembró una superficie de $3 \mathrm{~m}^{2}$ y regularmente cuida su huerto. 
Figura 6. Actividades del mes de diciembre de 2017
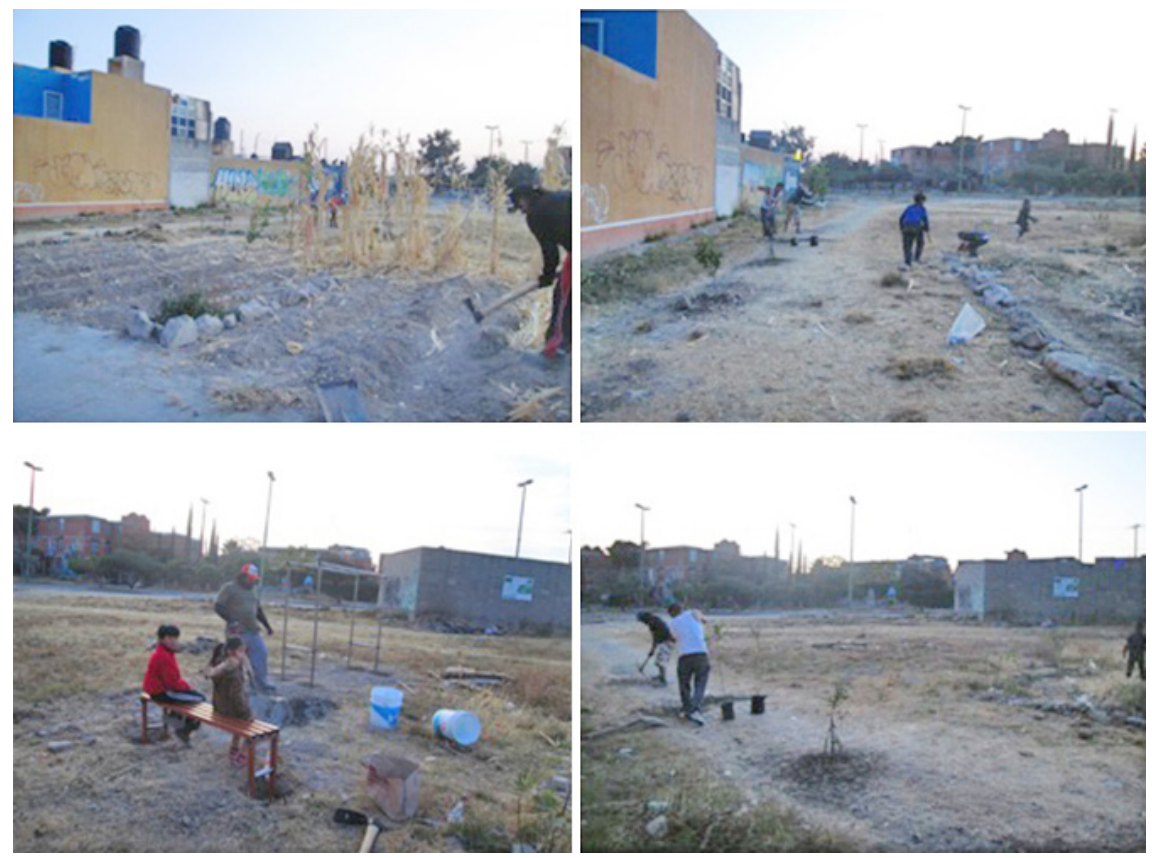

Fuente: elaboración propia.

Figura 7. Mantenimiento de árboles y control de plantas
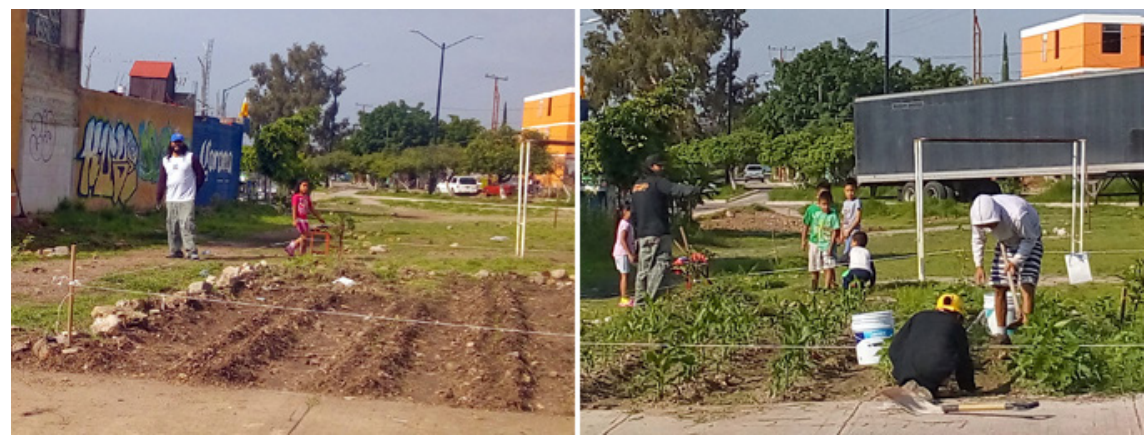

Fuente: elaboración propia. 
Figura 8. Excavado de cepas para árboles

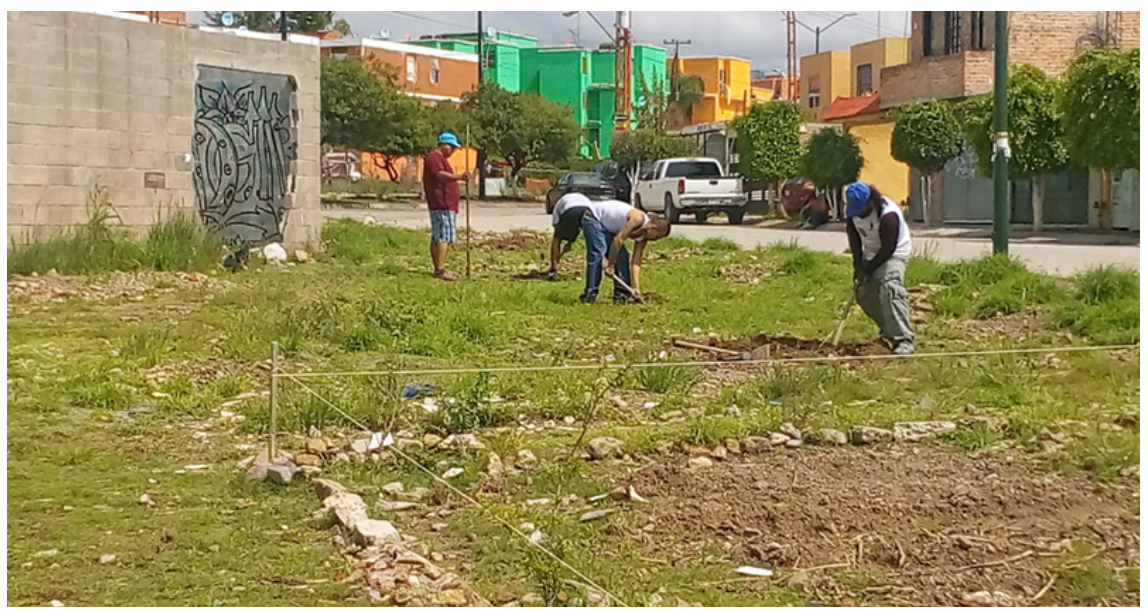

Fuente: elaboración propia.

Figura 9. Huerto implementado por niños
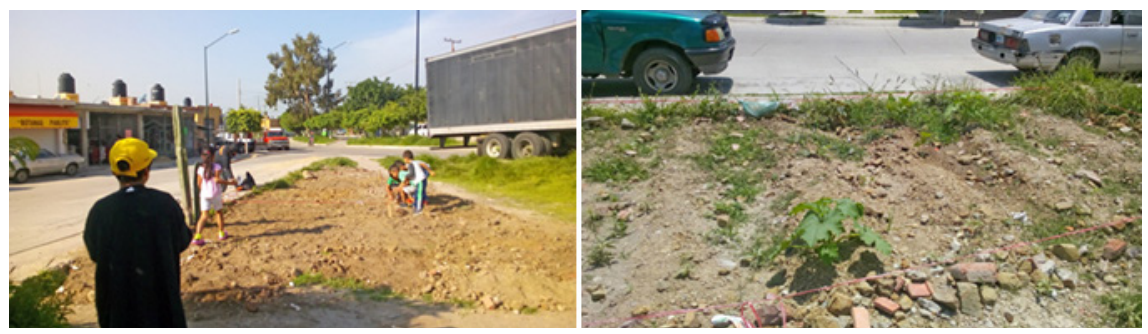

Fuente: elaboración propia.

\section{Resultados del modelo}

En la interface de Netlogo (figura 10), los agentes de color violeta representan a los vecinos de la colonia; los agentes de color rojo, a los contaminadores, ${ }^{8} \mathrm{y}$ un agente verde representa al apropiador. En este modelo, centramos nuestra atención en el mecanismo de aprendizaje. Suponemos que si los vecinos se apropian del área de donación como si fuera ajena a través de un proceso de aprendizaje, pueden apropiársela como si fuera propia. De esta manera, hemos establecido dos parámetros: el primero corresponde a un reforzamiento positivo - si un agente (vecino) en el mundo artificial mira al agente apropiador, aumenta en 0,84 su tasa de aprendizaje y empieza a apropiarse del espacio disponible-, y el segundo a un reforzamiento negativo, ya que

8. En este modelo, los contaminadores no tienen la capacidad de aprender, sino solo de contaminar. 
Figura 10. Interface Netlogo

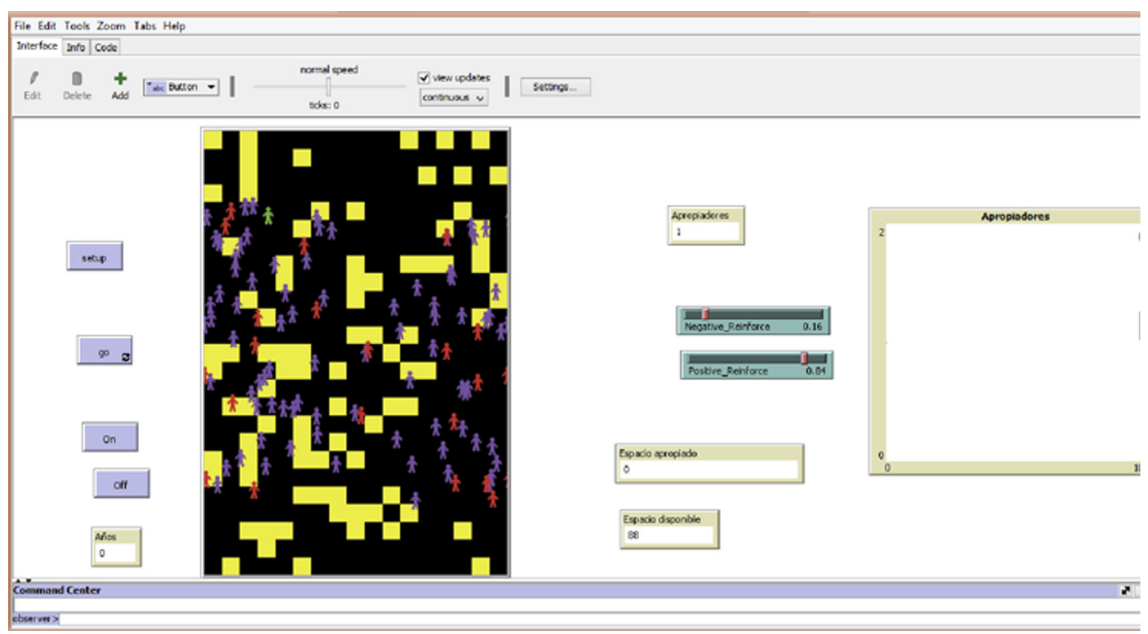

Fuente: elaboración propia.

cuando deja de verlo y extingue su aprendizaje en 0,16 , y si su nivel de aprendizaje es menor a 0,84 , aprende que el espacio es ajeno y deja de apropiarse del espacio disponible.

Simulamos 10 veces el modelo con diferentes condiciones iniciales. La frecuencia de agentes que aprenden a apropiarse del espacio fue de dos, tres y más de cuatro. De promedio, obtuvimos que 121 días al año dos agentes se están apropiando del espacio, 40 días tres agentes lo hacen, y 10 días cuatro agentes (tabla 1).

Tabla 1. Frecuencia de agentes apropiadores

\begin{tabular}{|c|c|c|c|}
\hline Ensayo & Frecuencia de 2 & Frecuencia de 3 & Frecuencia $=>4$ \\
\hline 1 & 72 & 1 & 0 \\
\hline 2 & 150 & 44 & 8 \\
\hline 3 & 91 & 17 & 2 \\
\hline 4 & 146 & 82 & 28 \\
\hline 5 & 142 & 33 & 14 \\
\hline 6 & 112 & 44 & 7 \\
\hline 7 & 119 & 36 & 5 \\
\hline 8 & 127 & 44 & 12 \\
\hline 9 & 129 & 54 & 10 \\
\hline 10 & 122 & 47 & 15 \\
\hline$\sum$ & 1210 & 402 & 101 \\
\hline $\mathrm{X}$ media & 121 & 40,2 & 10,1 \\
\hline $\mathrm{P}$ & 0,34 & 0,11 & 0,03 \\
\hline
\end{tabular}

Fuente: elaboración propia. 
Figura 11. Simulaciones

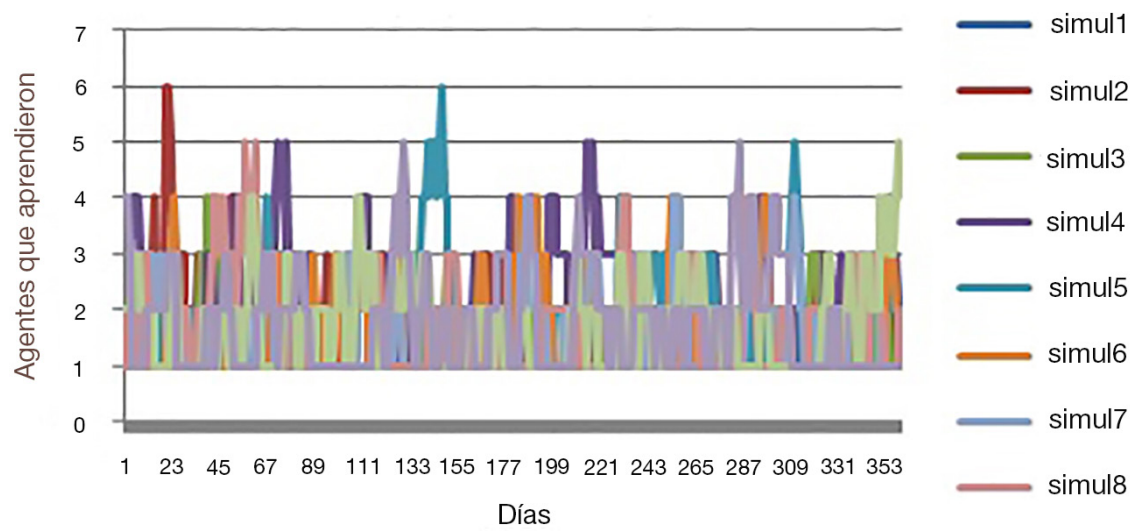

Fuente: elaboración propia.

Como podemos apreciar en la figura 11, el rango de agentes que se apropian del espacio osciló de dos a cuatro. A pesar de que observamos algunos casos de cinco y seis agentes, la mayor frecuencia fue de dos agentes. Cabe mencionar que la gráfica comienza en uno y no en cero, porque desde el inicio de la simulación existe un agente apropiador.

La tasa de apropiación de aprendizaje que empleamos fue obtenida de un estudio, como ya mencionamos (tabla 2).

Tal como dijimos, determinamos los valores de 0,84 para la tasa de aprendizaje y de 0,16 para la de olvido, porque nos permitieron describir el fenómeno empírico de apropiación. Sin embargo, como sugieren Behrens et al. (2007), establecer dichos valores sigue siendo tema de debate en el ámbito científico. En este sentido, en nuestro caso de estudio observamos que en el primer ensayo los niños aprendieron a apropiarse del espacio. Además, pasado dicho ensayo, su curva de aprendizaje describió un comportamiento asintótico. Es decir, más ensayos no generaban más aprendizaje. De esta manera, establecimos que la diferencia entre la novedad $-\beta-$ y el error de predicción $-\lambda$ - fue de 0,16 , lo cual nos da una tasa de aprendizaje de 0,84 en el primer ensayo, una tasa

Tabla 2. Modelo de Rescorla-Wagner (1972)

\begin{tabular}{crrrrr}
\hline Ensayo & \multicolumn{1}{c}{$\Delta \mathbf{v}$} & $\mathbf{V t}$ & $(\lambda-\mathrm{Vt})$ & $\alpha \beta(\lambda-\mathrm{Vt})$ & \multicolumn{1}{c}{$\Delta \mathbf{v}$} \\
\hline 1,0 & 0,0 & 0,0 & 100,0 & 84,0 & 84,0 \\
2,0 & 84,0 & 84,0 & 16,0 & 14,9 & 14,9 \\
3,0 & 14,9 & 98,9 & 1,1 & 1,1 & 1,1 \\
4,0 & 1,1 & 100,0 & 0,0 & 0,0 & 0,0 \\
5,0 & 0,0 & 100,0 & 0,0 & 0,0 & 0,0 \\
\hline
\end{tabular}

Fuente: elaboración propia 
Figura 12. Curva de aprendizaje

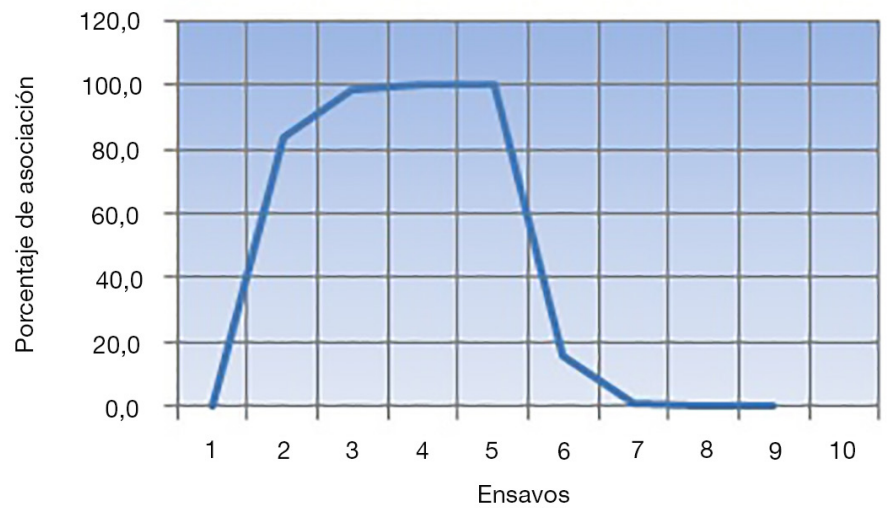

Fuente: elaboración propia.

de 14,9 en el segundo, hasta que el aprendizaje es cero, es decir, la diferencia entre la sorpresa y la predicción son cero (tabla 2). De igual manera, podemos observar en la tabla 2 que la fuerza asociativa - Vt - en el primer ensayo es 0 , y en el segundo es 0,84 . Por lo que deducimos que la tasa de reforzamiento negativo $-\lambda-\mathrm{Vt}$ - es de 0,16 . Con esto en mente, construimos la curva que describe el aprendizaje y la extinción en el modelo basado en agentes descrito arriba (figura 12). Cabe mencionar que en el modelo de Rescorla-Wagner (1972) el aprendizaje nunca llega al cien por ciento, y la curva describe un comportamiento asintótico.

\section{Análisis y discusión}

El ambiente social se puede conceptualizar como el tipo de interacciones que establecen las personas con su entorno (Granada, 2001). Además, dichas interacciones están influenciadas por los roles y las actividades que realizan las personas en dichos entornos (Granada, 2001). En este sentido, en nuestro caso de estudio existía el reconocimiento por parte de los vecinos de que el área de donación era ajena hasta antes de los procesos de apropiación con agricultura que llevamos a cabo. El reconocimiento explícito por parte de los vecinos de que el área de donación es propia después de dichos procesos nos sugiere que se ha experimentado un proceso de apropiación de un espacio ajeno a un espacio propio. Dicho proceso es, en suma, complejo, y consideramos que el modelo que hemos empleado en su estudio, basado en agentes, pese a utilizar mecanismos deterministas, corresponde en este documento siempre «a un conjunto complejo de fenómenos, y no a un fenómeno simple y directamente observable» (Lazarsfeld y Boundon, 1985). Además, reconocemos que los parámetros usados en dicho modelo podrían «variar considerablemente según el medio social del individuo o individuos estudiados» (Lazarsfeld y Boundon, 1985). Lo 
que nos interesa exponer en las siguientes líneas es cómo interpretar el proceso de apropiación del espacio como un proceso de aprendizaje condicionado, y en qué medida el modelo basado en agentes que hemos desarrollado ayuda a explicar dicho fenómeno.

Primero, como sugiere Durkheim (2001), los actos que realizamos están definidos fuera de nosotros en el derecho y las costumbres, y, a pesar de eso, dicha realidad no deja de ser objetiva porque no soy yo quien los ha creado, sino que los he recibido por medio de la educación. De esta manera, los modos de actuar, de pensar y de sentir presentan la propiedad notable de que existen fuera de las conciencias individuales (Durkheim, 2001). Además, dichas conductas o pensamientos no son solo exteriores al individuo, sino que están dotados de un poder imperativo y coercitivo en virtud del cual se imponen a él, lo quiera o no (Durkheim, 2001).

En este sentido, en las charlas que hemos tenido con los vecinos de la colonia, principalmente antes de implementar la apropiación con agricultura y al finalizar el primer año de labores, se nos comunicó que no querían participar en el proyecto por temor a confrontarse con otros vecinos y por temor a que el Gobierno tomará alguna acción legal contra ellos, ya que sentían que apropiarse de este espacio era un acto ilícito, al ser un área de donación de la colonia.

Así pues, podemos argumentar que muchos de los valores y fines que parecen orientar la acciones de las personas no los podemos comprender a menudo con plena evidencia, sino tan solo en ciertas circunstancias, captarlos intelectualmente (Weber, 2002). De esta manera, podemos interpretar que el método científico construye y expone las conexiones — subjetivas aprendidas condicionalmente (cultura) - del comportamiento humano que influyen en su acción (Weber, 2002). Dicha acción se estudia como la desviación de la acción puramente racional con un fin bien definido; la subjetividad se estudia científicamente como una desviación de un fin puramente racional (Weber, 2002).

De este modo, los resultados empíricos que mostramos de la apropiación del espacio con agricultura en un contexto urbano son una desviación en el curso racional de la construcción de las ciudades, donde el Gobierno se encarga de administrar y cuidar el territorio. Por el contrario, esta desviación permite la apropiación subjetiva del espacio en condiciones donde el espacio es ajeno, por lo que consideramos que a través de nuevos aprendizajes podemos lograr una transformación en la forma en que concebimos la realidad. En este plano, el modelo de aprendizaje asociativo de Rescorla-Wagner (1972) se basa en la teoría pavloviana, que tiene como punto central identificar una respuesta y un estímulo no condicionado - el ejemplo clásico es el de la salivación. En presencia de alimentos, las glándulas salivales se activan sin necesidad de ningún tipo de adiestramiento anterior. A esto se le llama fuerza asociativa entre un estímulo neutral y una respuesta incondicionada (Rescorla y Wagner, 1972). De esta manera, suponemos que un estímulo neutral es el espacio y que la respuesta incondicionada es apropiárselo. Como argumento lógico, establecemos que si A es un organismo que ocupa un lugar en un espacio y tiempo 
definido, y que $B$ es otro organismo en el mismo tiempo definido, entonces A y $B$ no pueden ocupar el mismo espacio al mismo tiempo. Es decir, toda vez que un espacio esté disponible puede ser ocupado. ${ }^{9}$ Por ejemplo, si soy expuesto a ver a personal de limpieza en un área de donación, cada vez que los observe aprendo que esa área pertenece al municipio, que es ajena y que no es mi responsabilidad cuidarla. Reconocemos que hablamos de procesos dinámicos complejos y adaptativos, que incorporan tanto actitudes personales como roles institucionales de la Administración pública, y que cuando ambos se unen generan normas de conducta (Hofstede, 2018). Resaltando que son procesos dinámicos adaptativos, podemos orientarlos en alguna dirección a través de aprendizaje asociativo.

En resumen, si en ensayos sucesivos percibo el espacio como ajeno, es decir, está ocupado o es de alguien más, la apropiación del espacio que lleve a cabo será como un contenedor de residuos. Por ejemplo, cuando separamos la basura, e incluso cuando la depositamos en contenedores especiales, nos estamos apropiando de un espacio ajeno, los rellenos sanitarios. Sin embargo, debido a que no lo observamos directamente ante nuestros ojos, es como si la basura desapareciera. Concretando, el estímulo neutral es el espacio y la respuesta incondicionada es apropiárselo. De esta manera, si ensayamos con un estímulo condicionado donde el espacio aparece ante nuestros ojos como ajeno, y con una respuesta condicionada donde el espacio ajeno sirve para depositar basura, después de varios ensayos habremos aprendido que en presencia de un espacio ajeno la apropiación es como contenedor de basura. Por el contrario, si cambiamos la respuesta ante el estímulo neutral, es decir, si exponemos a los vecinos de la colonia a ensayos en los que un agente ocupa el espacio ajeno con cultivos, plantas y árboles, y además dicho agente limpia periódicamente el área de donación, los vecinos aprenden con cada ensayo que el espacio es propio, y que ellos también se lo pueden apropiar. De esta manera, se reduce la apropiación con basura y escombro. Por lo que sugerimos que el modelo basado en agentes que hemos construido es útil en el estudio de la apropiación del espacio como aprendizaje condicionado. Los parámetros establecidos pueden ser ajustados según la tasa de aprendizaje que sea determinada.

$\mathrm{Al}$ respecto, los resultados simulados muestran que dos agentes es la frecuencia más probable de personas que se apropiarán del espacio. Dichos resultados son similares a los obtenidos empíricamente. Sin embargo, a la fecha, dichos resultados son exploratorios. El modelo basado en agentes que discutimos permite ajustar los parámetros de tasa de aprendizaje y extinción. En particular, este es un tema importante, porque en los modelos de condicionamiento clásico con el modelo de Rescorla-Wagner (1972) las tasas de aprendizaje y extinción son fijas. Por ejemplo, en el modelo presentado por Eptein (2013), la tasa de aprendizaje y olvido es fija para todos los agentes. Y aunque esto nos facilita estudiar los procesos de aprendizaje asociativo, es necesario imple-

9. Sin embargo, en el plano subjetivo pueden existir apropiaciones simultáneas del espacio (Jirón e Iturra, 2011). 
mentar un mecanismo que permita establecer diferentes distribuciones de tasa de aprendizaje y extinción en los agentes. Es decir, crear condiciones de heterogeneidad. De esta manera, podremos identificar si los resultados del modelo se sostienen, lo cual nos permitiría tener evidencia a favor o en contra de establecer valores fijos de la tasa de aprendizaje y extinción. Por último, señalamos que los resultados de las simulaciones del modelo presentado son relevantes desde un punto de vista metodológico, es decir, que podemos emplear un enfoque que incorpore tanto evidencia empírica como resultados simulados. Sin embargo, una crítica que se hace a este tipo de estudios, en los que se usan los datos empíricos para generar un modelo de simulación, es que las respuestas emergentes del modelo pueden considerarse respuestas impuestas (Railsback, 2001). No obstante, este enfoque es un avance en los estudios de validación de los modelos basados en agentes.

\section{Conclusiones}

Podemos establecer que el modelo del aprendizaje asociativo de Rescorla-Wagner (1972) es útil en el estudio de la apropiación del espacio como aprendizaje condicionado. Además, existe una relación positiva entre el aprendizaje asociativo y la apropiación del espacio. De esta manera, se pueden ensayar procesos de aprendizaje asociativo donde el espacio ajeno sea percibido como un espacio propio. En nuestro trabajo, dicho aprendizaje se logró con agricultura en un periodo de dos años. La tasa de aprendizaje de 0,84 usada en el modelo arrojó resultados de simulaciones similares a los obtenidos en el estudio empírico.

\section{Agradecimientos}

A todo el equipo del Laboratorio de Simulación de Dinámicas Socio-Históricas (LSDS) de la Universidad Autónoma de Barcelona. En especial, agradezco al Dr. Francisco J. Miguel Quesada su valiosa ayuda durante mi estancia de investigación sobre modelos de simulación basados en agentes. También agradezco al Dr. Ricardo Valdez Cepeda y al Dr. Irineo López Cruz de la Universidad Autónoma Chapingo su valioso apoyo y comentarios aportados a este trabajo de investigación.

\section{Financiación}

Agradezco al Consejo Nacional de Ciencia y Tecnología (CONACYT) la beca que me ha permitido sufragar esta investigación.

\section{Referencias bibliográficas}

Behrens, T.; Woolrich, Mark; Walton, M. y Rushworth, M. (2007). «Learning the value of information in an uncertain world». Nature Neuroscience, 10 (9). <https://doi.org/10.1038/nn1954> 
Black, A. y Prokasy, W. (1972). Classical Conditioning II: Current Theory and Research. Appleton-Century-Crofts. Nueva York: Educational Division, Meredith Corporation. Disponible en:

<https://www.researchgate.net/publication/233820243_A_theory_of_Pavlovian_conditioning_Variations_in_the_effectiveness_of_reinforcement_and_nonreinforcement/link/00b495321aae3132e8000000/download>.

Durkheim, Emile (2001). Las reglas del método sociológico. Fondo de Cultura Económica.

Epstein, Joshua M. (2013). Agent-Zero Toward Neurocognitive Foundations for Generative Social Science. Princeton University Press.

Epstein, J. y Axtell, R. (1996). Growing artificial societies. Social Science from the Bottom up. The Brooking Institute.

FARAGó, László (2016). «The social (sociological) turn in the discourse about space». Romanian Review of Regional Studies, XII (1). Disponible en: <http://rrrs.reviste.ubbcluj.ro/arhive/Artpdf/v12n12016/RRRS12120161.pdf>.

Fontana-Giusti, G. (2016). The Unconscious and space Venice and the work of Albrecht Durer. En: Shannon, H. J. y Lorens, E. H. Architecture and the Unconscious. Imprint Routledge.

FraAssen, Bas C. van (1970). An introduction to the philosophy of time and space. Random House.

García-VAldecasas, José I. (2011). «La simulación basada en agentes: una nueva forma de explorar los fenómenos sociales». Reis, 136, 91-110. <https://doi.org/10.5477/cis/reis.136.91>

Gershenson, Carlos (2007). «Design and control of self organizing systems». CopIt ArXives.

Granada, Henry (2001). «El ambiente social». Investigación y desarrollo, 9 (1), 388407. Universidad del Norte Barranquilla, Colombia. Disponible en: <http://www.redalyc.org/pdf/268/26890102.pdf>.

Hofstede, Gert Jan (2018). «Artificial sociality». Disponible en <https:/geerthofstede.com/wp-content/uploads/2018/05/Artificial-sociality2018-05-22-Web-version.pdf>.

Jirón, P. e Iturra, Luis (2011). «Momentos Móviles. Los lugares móviles y la nueva construcción del espacio público». Arquitecturas del Sur (39), 44-57. Disponible en <https://www.researchgate.net/publication/220027019_Momentos_Moviles_Los_ lugares_moviles_y_la_nueva_construccion_del_espacio_publico $>$.

LazArSFeld, Paul F. y Boudon, Raymond (1985). Metodología de las ciencias sociales, de los conceptos a los indices empíricos. Barcelona: Editorial Laia.

Lehrer, Jonah (2009). How we decide? Boston-Nueva York: Hougton Mifflin Harcourt.

Loukaki, Argyro (2014). The geographical Unconscious. Routledge.

Mazoyer, M. y RoudArT, L. (2006). A history of world agriculture. Londres: EARTHSCAN.

Noschis, K.; Dosio, Marie-José; Feddersen, Pierre; Trianis, Euphrosyne (1978). "Appropriation of space: a method and two case studies». Ekistics, 45 (273). $<$ https://www.jstor.org/stable/43623631?seq=1>.

ONU (2016). Conferencia de las Naciones Unidas sobre la Vivienda y el Desarrollo Urbano Sostenible (Hábitat III). Nueva Agenda Urbana.

ONU-Haвitat (2016). «Reporte ciudades del mundo. Urbanización y Desarrollo: futuros emergentes». 
Railsback, S. (2001). "Concepts from complex adaptive systems as a framework for individual-based modeling». Ecological Modelling, 139, 47-62. <https://doi.org/10.1016/S0304-3800(01)00228-9>

Vidal, Tomeu y Pol, Enric (2005). «La apropiación del espacio: una propuesta teórica para comprender la vinculación entre las personas y los lugares». Anuario de Psicología, 36 (3), 281-297. Universitat de Barcelona. Disponible en: <https://www.raco.cat/index.php/AnuarioPsicologia/article/view/61819>.

Vidal, Tomeu; Pol, Enric; Guàrdia, Joan; Peró, Maribel (2004). «Un modelo de apropiación del espacio mediante ecuaciones estructurales». Medio Ambiente y Comportamiento Humano, 5 (1 y 2), 27-52. Disponible en: <https://mach.webs.ull.es/PDFS/Vol5_1y2/VOL_5_1y2_b.pdf>.

Weber, Max (2002). Economía y sociedad. Fondo de Cultura Económica.

Wilensky, U. y Rand, William (2015). An introduction to Agent-Based Modeling. Modeling Natural, Social and Engineered complex systems with Netlogo. MIT Press.

Wilson, W. J. (2012). «The Rescorla-Wagner Model, Simplified». Albion College. Disponible en: <http://webcache.googleusercontent.com/search?q=cache:n-4iMzqS848J:campus.albion.edu/wjwilson/files/2012/03/RWSimplified. $\mathrm{pdf}+\& \mathrm{~cd}=3 \& \mathrm{hl}=\mathrm{es} \& \mathrm{ct}=\mathrm{clnk} \& \mathrm{gl}=\mathrm{mx}>$. 





end

to move-vecinos

ask vecinos[fd 1$]$

ask vecinos

[ if can-move? 1 [

if any? agricultors-on patch-ahead 1 [set energy energy + Positive_Reinforce ]

if energy $>=0.8$ [set color green ]]]

ask vecinos[

if $($ color $=$ green $)$ and $($ energy $>=0.8)$ and (use = "apropiable") [set pcolor green]]

ask vecinos

[if energy $>0.8$ [ set energy energy - Negative_Reinforce

if energy $<0.8$ [set color violet] ] 